\title{
Metodologías de innovación docente: la perspectiva de género en Comunicación Audiovisual $^{1}$
}

\author{
María Isabel MenÉNDEZ MENÉNDEZ \\ Universidad de Burgos \\ mimenendez@ubu.es
}

\begin{abstract}
Resumen
La inscripción de la universidad española en el Espacio Europeo de Educación Superior (EEES) conlleva una modificación del proceso de enseñanza-aprendizaje que, entre otras cuestiones, exige incorporar el principio de igualdad de oportunidades entre mujeres y hombres en los nuevos Grados. Sin embargo, la falta de recorrido histórico y el desconocimiento de contenidos, metodologías y materiales específicos, hace muy difícil la incorporación de este principio por parte de un personal docente que, con frecuencia, carece de formación y experiencia en la materia. El presente artículo ofrece claves metodológicas para integrar los aportes de los Estudios de las Mujeres, Feministas y de Género en las aulas de Comunicación Audiovisual.
\end{abstract}

Palabras clave: género; comunicación audiovisual; innovación; metodología docente; feminismo.

\section{Methodologies for Teaching Innovation: The Gender Perspective in Audiovisual Communication}

\begin{abstract}
The adscription of the Spanish universities to the European Higher Education Area implies changes in the teaching-learning process that, among other things, demand the incorporation of the principle of equality between the sexes in the new BA programs. Nevertheless, its short history and the scarce knowledge about contents, methods, and specific materials make this principle very difficult to apply for a teaching staff that very often lacks training and experience in the area. This paper offers some methodological clues for the incorporation of the contributions of the Women's, Feminist, and Gender Studies areas into the Audiovisual Communication classroom.
\end{abstract}

Key Words: gender; audiovisual communication; innovation; teaching methodology; feminism.

\section{Referencia normalizada:}

Menéndez Menéndez, M. I. (2013) Metodologías de innovación docente: la perspectiva de género en Comunicación Audiovisual. Historia y Comunicación Social. Vol. $18 \mathrm{~N}^{\mathrm{o}}$ Especial Octubre. Págs. 699-710.

\section{Sumario:}

1. Introducción. 2. La perspectiva de género y el EEES. 3. Metodología en los estudios de comunicación audiovisual. 3.1. Estrategias metodológicas: diseño curricular en comunicación. 3.2. Innovación y praxis: el caso del cine. 4. Conclusiones. 5. Bibliografía. 


\section{Introducción}

El Espacio Europeo de Educación Superior (EEES) y su adaptación a la universidad española ha exigido una completa modificación del proceso de enseñanza-aprendizaje. Entre las novedades que recogen los nuevos Grados verificados por las agencias de calidad se indica la necesidad de incorporar el principio de igualdad entre mujeres y hombres en todos los títulos. Ello se traduce en la obligatoriedad de que todo el alumnado universitario reciba formación en género (Bosch et al., 2011), aunque los diseños curriculares y los textos legales no explican cómo debe realizarse dicha incorporación, dejando en manos de docentes e instituciones superiores el diseño y aplicación de estas acciones.

Sin embargo, la metodología y los contenidos necesarios para velar por esta premisa no han formado parte del mainstream educativo, especialmente en el ámbito universitario. De hecho, las aportaciones de los Estudios de las Mujeres, Feministas y de Género ni siquiera son aceptadas por toda la comunidad educativa que, en ocasiones, incluso llega a discutir su carácter científico. Todo ello explica que el cuerpo docente se encuentre, con frecuencia, ante la dificultad de llevar a la praxis lo que exige el diseño curricular, cuando no con la angustia de desconocer casi todo de aquello que se le exige. Esta laguna también explica la ausencia de una metodología precisa y de materiales de apoyo.

Lo antedicho revela una realidad: aunque los estudios de género en la universidad española experimentaron un importante impulso a finales de los años noventa del siglo XX, es un hecho que, cuando está prácticamente culminada la implantación del nuevo modelo educativo en España, la bibliografía de referencia advierte que la perspectiva de género no sólo no ha sido incorporada de forma transversal, como hubiera sido lo deseable, sino que, en algunos aspectos, ha experimentado un retroceso. El trabajo de las académicas feministas no ha conseguido implantarse con normalidad en la Academia. Así, la mayoría de universidades han instaurado títulos de posgrado específicos (muchos de ellos, hoy en riesgo debido a la crisis económica) mientras que, paradójicamente, muchas materias que formaban parte de las extintas Licenciaturas o Diplomaturas han desaparecido de la oferta de Grados. De manera similar, el Gender Mainstreaming o transversalidad no se ha integrado, como revela la observación de las guías docentes que las universidades publican en sus páginas web para que sean consultadas por el alumnado.

Por todo ello, es necesario pensar y diseñar prácticas docentes innovadoras y originales, que permitan a profesorado y alumnado acceder a las competencias y conceptos básicos que se requieren en esta materia. En este sentido, el presente artículo ofrece algunas claves metodológicas para incorporar la perspectiva de género en los estudios de Comunicación Audiovisual. Para ello, se ofrecen principios esenciales sobre el enfoque de género y su aportación a las Ciencias Sociales. A continuación, se exponen cuestiones metodológicas que, de forma sintética, ofrecen claves que permiten incorporar en la práctica docente del título de Comunicación Audiovisual las aportaciones de los Estudios de las Mujeres, Feministas y de Género. 


\section{La perspectiva de género y el eees}

En la esfera de la praxis, la perspectiva de género es la metodología mediante la que incorporar la epistemología feminista junto a los aportes críticos que, relacionados con el sexo y el género, han elaborado diversas Ciencias Sociales, entre las que destaca la sociología, la antropología, la filosofía o la psicología entre otras. Básicamente se puede definir como una categoría analítica que permite diferenciar lo biológico de lo cultural y social; analizar y comprender las características y necesidades específicas de mujeres y varones; visibilizar las relaciones que se establecen entre hombres y mujeres, organizadas según un modelo de poder y limitadas por los roles de género; conocer la situación y posición que ocupan unos y otras en todos los ámbitos de la vida; reconocer la desigualdad histórica que soportan las mujeres; y visibilizar las aportaciones de éstas a la sociedad.

Por consiguiente, utilizar un enfoque de género consiste en adoptar una lectura crítica que permite (re)definir los principios que la sociedad ha dado por válidos y universales y que, sin embargo, se han revelado como discursos excluyentes. Esta lectura tiene un objetivo político: remover la discriminación y la desigualdad entre sexos, de ahí que las leyes actuales velen por su incorporación pero también que, en muchas ocasiones, existan resistencias a su aplicación. Es decir, esta nueva mirada, imprescindible hoy en las Ciencias Sociales, se basa en el estudio de las relaciones humanas y cómo éstas afectan a las vivencias de mujeres y hombres. El resultado es que cuestiona los saberes canónicos y plantea un nuevo orden científico.

Debido a la perspectiva androcéntrica que existe en nuestra sociedad, las mujeres han estado históricamente ausentes de todo protagonismo. Esta ausencia es un hecho en todas las disciplinas: la historia, el deporte, la política o la salud han funcionado con estándares masculinos que luego se han aplicado a las mujeres. Por ello, la visibilización de las relaciones que se establecen entre ambos sexos así como el análisis de la situación y posición que ambos ocupan, exige tener en consideración los diversos planos en que intervienen los agentes de socialización y los discursos que cada cultura sanciona como hegemónicos.

El análisis de género permite comprender e intervenir en la injusta jerarquía sexual que se concreta, entre otros problemas, en la negación y desprecio sobre las aportaciones femeninas a la sociedad, la discriminación salarial, la violencia de género o la ausencia de las mujeres del canon, esto es, de la producción de los discursos intelectuales o artísticos considerados prestigiosos. En el ámbito de los medios de comunicación, la cultura de masas y la cultura popular, la ausencia de las aportaciones femeninas es una evidencia demostrada desde hace décadas, por lo que los estudios universitarios de comunicación deben incorporar, más allá de la obligatoriedad de la ley, una parte de la realidad que hasta ahora ha sido expoliada. Por otro lado, también existe consenso respecto al papel que los medios de masas tienen en la transmisión de estereotipos de género, lo que urge a estos agentes a revisar sus preceptos y realizar una autocrítica que permita desvelar sus prácticas androcéntricas y/o sexistas. 
De acuerdo con Stimpson, existen tres formas básicas de incorporar la perspectiva de género en la docencia universitaria, las dos primeras mucho más desarrolladas en el caso de la universidad española. En primer lugar, mediante títulos específicos, esto es, la incorporación de programas de Estudios de las Mujeres, Feministas o de Género en la oferta formativa. En segundo lugar, a través de la inscripción de una asignatura específica dentro de un programa de un título no específico. Por último, y asumiendo la metodología del Gender Mainstreaming, integrando los contenidos en cualquier materia perteneciente a cualquier plan de estudios. Como han demostrado Bosch y Ferrer (2012), en la universidad española se pueden encontrar ejemplos del primero y segundo de los casos, especialmente en los estudios de posgrado, pero es complejo encontrar experiencias sobre la tercera de las posibilidades.

En efecto, en cuanto a la presencia de contenidos de estudios de género en la docencia universitaria, Bosch y Ferrer señalan que, en la mayoría de asignaturas troncales y obligatorias, no se incluyen contenidos que puedan considerarse propios de los estudios de género. Dichos contenidos aparecen (si lo hacen), casi en exclusiva en la oferta de asignaturas optativas y en los Títulos Propios de las universidades. Las autoras incluso perciben cierto retroceso respecto a los estudios anteriores al EEES (Licenciaturas y Diplomaturas) pues han desaparecido, con la implantación de los Grados, algunas materias de carácter optativo dedicadas al género. Bosch y Ferrer enfatizan que la presencia de esos contenidos depende casi siempre de la existencia y relevancia de grupos de investigación, seminarios o institutos dedicados a los Estudios de las Mujeres, Feministas y de Género en las universidades. Es decir, no se ha conseguido la normalización de dichas materias y su enseñanza depende, en esencia, de la voluntad y formación específica de cada docente.

Teniendo en cuenta, por tanto, que la práctica de asumir la igualdad como principio rector es más intencional que estructural y que el diseño de títulos oficiales no es responsabilidad de quienes enseñan, no deja de ser curioso que sea la tercera de las posibilidades señaladas por Stimpson la que menos éxito ha tenido. Ello reafirma la tesis que se planteaba al principio. Si bien es posible que el profesorado carezca de motivación, lo cierto es que la mayoría de docentes no dispone de los instrumentos necesarios para incorporar el género de forma transversal, básicamente porque carece de formación específica. Si bien esta capacitación sigue siendo necesaria, y debería ser asumida por las universidades en sus planes de formación docente, las siguientes líneas intentarán arrojar un poco de luz respecto al cómo se hace, sugiriendo líneas metodológicas que ayuden al profesorado a impartir las materias de Comunicación Audiovisual con perspectiva de género.

\section{Metodología en los estudios de comunicación audiovisual}

La aplicación práctica de la perspectiva de género es un proceso en el que hay que reconocer separadamente la diferencia sexual y las atribuciones, ideas, represen- 
taciones y prescripciones sociales que se construyen tomando como referencia esa diferencia sexual (Lamas, 1995). El género es una forma de ordenación social que atribuye a hombres y mujeres roles, papeles y comportamientos diferenciados y es necesario volver a pensarlos para eliminar los estereotipos y la discriminación. En este sentido, los medios de comunicación y la industria audiovisual tienen una gran responsabilidad, por su capacidad de influencia y su alto nivel de penetración. De ahí la importancia de que el alumnado que en el futuro trabajará en estas industrias incorpore una perspectiva crítica y una posición activa ante la falta de equidad, algo que la metodología específica de género permite introducir.

Utilizar el enfoque anterior en la práctica profesional de las industrias culturales, o en lo que nos ocupa, en su enseñanza en las facultades de comunicación, implica un ejercicio que obliga a volver a pensar diversas cuestiones, todas ellas importantes. En primer lugar, es necesario contemplar a las mujeres y a los hombres desde todos los mensajes que se elaboran en las empresas mediáticas y audiovisuales. Por otro lado, hay que (re)elaborar materiales y contenidos docentes que no perpetúen el androcentrismo y la invisibilidad de las mujeres. Ello exige, por supuesto, discutir el canon, pero también un gran esfuerzo de búsqueda de nuevos documentos y fuentes.

Como hemos escrito en otro lugar, para ello habrá que tener en cuenta el protagonismo de las mujeres (para terminar con la invisibilidad), el uso de lenguaje no sexista (para nombrar a las mujeres como sujeto-activo), la incorporación de mujeres como fuente de información o protagonismo (para restaurar la legitimidad que les corresponde), la inclusión de temas y espacios tradicionalmente invisibles (para romper con las dicotomías hombre/mujer), la detección de imágenes o contenidos sexistas en los mensajes (para contribuir a paliar la discriminación, generando una opinión pública favorable a la igualdad), la atención a las problemáticas sociales que existen en función del género desde una óptica que las considere como temas políticos y tan relevantes como los que ocupan la agenda pública convencional (violencia sexista, conciliación de vida profesional y personal, etc.) y la exclusión de temáticas o tratamientos que perpetúan los estereotipos de género (Menéndez, 2007a: 149-168; Menéndez, 2007b: 81).

Hay que insistir en el hecho de que la práctica audiovisual y su enseñanza están recorridas, como otros discursos sociales, por rutinas androcéntricas que han acostumbrado a profesionales y audiencia a invisibilizar a las mujeres sin que ello parezca extraño o despierte reticencias. Es útil recordar que la poca o nula presencia de nombres de mujeres "acentúa el desinterés, refuerza el desconocimiento y crea una imagen imprecisa y desdibujada de este colectivo” (García, 2013: 784).

\subsection{Estrategias metodológicas: diseño curricular en comunicación}

En el ámbito de la educación en medios y discurso audiovisual, el diseño curricular debe tener en cuenta, al menos, las siguientes dimensiones:

1. Formación básica en conceptos relacionados con la igualdad entre hombres y mujeres: género, discriminación, paridad, feminismo, patriarcado, androcen- 
trismo, sexismo... son conceptos básicos pero que no forman parte del mainstream educativo y que muchas personas no pueden definir con corrección. El alumnado debe conocer esta terminología y, además, ser capaz de reflexionar sobre ella, poniéndola en relación con el panorama audiovisual y la actualidad. La ausencia de estos contenidos en la formación explica que, con frecuencia, se utilicen de forma errónea determinados conceptos que, sin embargo, cualquier persona con educación superior debería manejar correctamente. Además, la complejidad de los estudios de género exige que estos términos se expliquen contextualizados y acompañados de literatura especializada.

2. Aproximación a la historia de la lucha por los derechos de las mujeres con especial referencia al panorama audiovisual y/o artístico cuando es posible: hitos históricos, relación con los medios, agenda pendiente, etc. Es frecuente que el alumnado universitario desconozca casi todo sobre la diferencia histórica en la adquisición de los derechos de los hombres y de las mujeres, por lo que apenas conocen algo más que la existencia del sufragismo. Paliar esta laguna histórica es imprescindible si deseamos que salga de nuestras aulas con una perspectiva realmente universal (especialmente importante en un alumnado que se dedicará, en el futuro, a la comunicación). Ello permite, además, una reflexión crítica sobre las dificultades de las creadoras y pensadoras para acceder a la industria audiovisual y, especialmente, a la creación artística.

3. Discusión y reflexión sobre binomios esencialistas como lo femenino/masculino, su jerarquía e importancia social, su representación en la industria audiovisual y las posibles consecuencias en los públicos. Se trata de un trabajo analítico y reflexivo, que exige del alumnado un trabajo autónomo, maduro y no coercitivo. Es esta una labor perfecta para la metodología del taller y el trabajo grupal, por lo que se adapta perfectamente a los principios rectores del proceso de enseñanza-aprendizaje del EEES. El alumnado debe poder realizar un ejercicio crítico a partir de las herramientas intelectuales que ha recibido, relacionando conceptos y materiales, y reflexionando tanto individual como grupalmente sobre las cuestiones esenciales.

4. Conocimiento y reflexión sobre la aportación de las mujeres a la industria audiovisual e incorporación de las biografias relevantes. Es necesario rescatar la historia de las aportaciones femeninas en sus diferentes facetas y periodos históricos pues, aunque ha habido mujeres cuyas contribuciones al audiovisual han sido importantes, apenas son conocidas y, si lo son, no suelen formar parte del temario. Además, no es infrecuente que las pocas biografías femeninas que se llevan a las aulas aparezcan en función de estereotipos sexistas u otras lecturas sesgadas, empañando la importancia de sus carreras o negando los aspectos científicos y técnicos.

5. Incorporación de textos alternativos a los canónicos (que debido al androcentrismo son casi siempre masculinos), tanto escritos como audiovisuales. Estas lecturas y visionados deben preocuparse por recoger tanto la historia y 
aportaciones artísticas, técnicas o científicas de las mujeres como el pensamiento e investigación desarrollado por las académicas que las han rescatado del olvido. Desde hace décadas, el trabajo desde los Estudios de las Mujeres, Feministas y del Género ha elaborado un canon alternativo, muy rico y complejo, que permite superar la invisibilidad femenina en las bibliografías, lecturas y visionados que se proponen al alumnado, ahora más que nunca con la metodología de tipo práctico que exige el EEES.

6. Comprensión e información sobre las rutinas netamente sexistas que se producen en el mundo audiovisual en sus diferentes contextos. Tanto el androcentrismo como cierta tolerancia hacia el sexismo (cuando no el machismo explícito) permiten que, en general, no se reflexione especialmente ante discursos y mensajes que perpetúan la discriminación y que, incluso, pueden llegar a justificarse en nombre de la creatividad, la originalidad, el humor o el mismo mercado. Es imprescindible proveer al alumnado de herramientas intelectuales que les permitan identificar el sexismo y sus consecuencias, de forma que pueda adoptar una actitud activa para su erradicación. Hay materias de Comunicación Audiovisual con especial relevancia en este aspecto: la publicidad o la ficción son algunas de ellas.

7. Aproximación al marco legal que, en cuanto a la igualdad, afecta al contenido de los medios de comunicación y las industrias culturales. Así como el alumnado de Comunicación Audiovisual recibe nociones básicas sobre derecho a la información, debe conocer la legislación específica sobre igualdad, especialmente en lo que afecta a los medios de comunicación (Ley Orgánica 3/2007 para la Igualdad Efectiva de Mujeres y Hombres; legislación sobre publicidad; Ley Orgánica 1/2004 de Medidas de Protección Integral contra la Violencia de Género, etc.), así como los mecanismos de intervención para luchar contra la discriminación (códigos deontológicos para eliminar el sexismo; Observatorios de publicidad sexista, autorregulación publicitaria, etc.).

Concretando lo antedicho, el alumnado de Comunicación Audiovisual debe recibir, en el apartado de contenidos docentes, una información básica sobre mujeres protagonistas del sistema audiovisual, aportaciones de éstas a los diferentes discursos, bibliografía escrita por mujeres, contribuciones científicas de teóricas y expertas en comunicación así como dimensiones del sexismo explícito que recorre los medios de comunicación y el mundo audiovisual (publicidad, cine, periodismo, etc.).

Recordemos, asimismo, que se hace necesario que todas las personas con formación universitaria reciban una mínima formación en género (Bosch et al., 2011: 53). Por consiguiente, tendrán que conocer al menos el vocabulario especializado, eliminando errores frecuentes que luego aparecen en los mensajes mediáticos y que son fruto de la ausencia de formación e, incluso, de los prejuicios.

Los hombres y mujeres que se dedicarán a la comunicación en el futuro deben reflexionar y aprender, al menos, en dos planos: en primer lugar, en la capacitación para la eliminación del androcentrismo y la violencia simbólica. En segundo lugar, en 
la adquisición de contenidos básicos sobre la disciplina en relación con las mujeres. Esta última dimensión se concreta, como mínimo, en conocer los nombres de mujeres relevantes y su contribución científica, técnica o artística; detectar las necesidades y/o dificultades en dicha disciplina desde la perspectiva de género y familiarizarse con las obras críticas sobre la materia.

En cuanto a las capacidades y competencias que deben desarrollar, como se ha recogido ya, deben ser capaces de detectar el androcentrismo y el sexismo de manera autónoma y activa. Asimismo, tendrán que incorporar estrategias de eliminación de dichas problemáticas, incluyendo el uso del lenguaje y la lectura crítica ante imágenes o contenidos violentos desde el punto de vista simbólico.

\subsection{Innovación y praxis: el caso del cine}

El estudio del cine, una parte muy importante de los estudios de Comunicación Audiovisual, no escapa al diagnóstico reflejado en las líneas anteriores. Sin embargo, existe una amplia bibliografía sobre teoría fílmica feminista. A partir del ya clásico texto de Laura Mulvey (1988), este corpus científico experimentó un gran impulso a partir de los años setenta del siglo pasado. El trabajo de las académicas ha reflexionado, por ejemplo, sobre los códigos de representación y los símbolos que construyen significados relacionados con hombres y mujeres, pero también ha rescatado del olvido y la indiferencia la obra de las creadoras en el ámbito cinematográfico.

Aunque no aparecen en la historia canónica del denominado séptimo arte, las mujeres han sido numerosas en la industria cinematográfica desde su nacimiento. Ha habido realizadoras, creadoras, técnicas... algunas (o muchas) de ellas preocupadas por elaborar un discurso alternativo al hegemónico, en el que las mujeres apenas son algo más que un objeto de seducción. Siguiendo el esquema propuesto en el epígrafe anterior, estas serían algunas cuestiones que deberían formar parte de la enseñanza en el título de Comunicación Audiovisual:

Conocimiento de los nombres de mujeres relevantes en la disciplina y su contribución científica, técnica o artística: las mujeres están en el cine desde sus inicios. Así, pioneras como Alice Guy (1873-1968), que rodó su primera película en 1896 (La Fée aux choux), deberían formar parte de la programación docente. La obra de Guy en concreto, como recoge García (2009: 24), es considerada por muchos textos como la primera película de ficción de la historia. Su obra fue muy prolífica y sus aportaciones fueron muchas: investigó sobre el coloreado en el cine, utilizó trucos cinematográficos, experimentó con la sonoridad, etc. García (2009: 25-32) aporta muchas otras propuestas de nombres que deberían acceder al aula: Lois Weber (1882-1939), Elvira Notari (1875-1946), Lotte Reiniger (1899-1981), Esther Shub (1849-1959), Dziga Vertov (1896-1954) o Elizaveta Svilova (1900-1975) por citas algunas. Obviamente, si ha habido mujeres importantes en los inicios del cine, las creadoras no han dejado de formar parte de su historia, por lo que su número no ha hecho más que aumentar con el tiempo y hasta la actualidad. Ello incluye también los talentos y creaciones de mujeres españolas que han conseguido destacar en época reciente, como Icíar Bollaín 
o Isabel Coixet entre otras. Descubrir sus biografías, obras y aportaciones es un reto que el profesorado debería defender pues añadir este conocimiento no resta, sino que suma al acervo científico.

Detectar las necesidades y/o dificultades en dicha disciplina desde la perspectiva de género: el cine es un discurso tan androcéntrico como el resto de producciones simbólicas, y la industria cinematográfica es tan sexista como la mayoría de organizaciones empresariales y/o laborales pues en ella se reproducen los problemas que tienen las mujeres en todos los ámbitos (segregación horizontal y vertical, empleo de menor calidad, dificultades de conciliación, etc.). Por ello, es importante analizar ambas esferas desde la perspectiva de género para descubrir, por ejemplo, las dificultades que tienen las creadoras para dirigir cine. Estas reflexiones deben llevarse al aula ya que invisibilizar esta realidad es ofrecer un panorama sesgado del cine y su actualidad.

La problemática de género en el audiovisual, tal y como recogen Arranz et al. (2009) en su estudio sobre el cine español entre 2000 y 2006, se concreta en que, en la empresa audiovisual, apenas existen mujeres en las posiciones de toma de decisión. Además, se reproduce el reparto de tareas en función de la división sexual del trabajo, por lo que ellas aparecen sobre todo en las labores de producción, apenas están en el grupo técnico (cámaras, sonido) y son mayoría en el grupo de especialistas (peluquería, vestuario, maquillaje). Por otro lado, apenas se ha incrementado el número de creadoras y realizadoras mientras que, en el mismo periodo, han aumentado las operas primas de nuevos directores varones; en cuanto a las ayudas a la creación, también presentan un fuerte desequilibrio (1 directora, 17 directores). Por otra parte, las mujeres apenas aparecen en los jurados de los festivales españoles y tampoco suelen ser premiadas en ellos.

En cuanto al análisis de las obras, las representaciones de género en los filmes siguen siendo androcéntricas y estereotipadas, otorgando poco protagonismo a las mujeres y perpetuando un discurso tolerante ante la violencia, tal y como explica Aguilar (2004). Para analizar estas variables es necesario recordar que el alumnado debe recibir formación básica sobre género, discriminación y sexismo, pues de otra manera no dispondrá de las claves analíticas precisas. El esfuerzo que las teóricas han realizado y que está plasmado en múltiples trabajos científicos, debe formar parte de las distintas asignaturas dedicadas al cine, pues su ocultación no favorece una enseñanza de calidad ni una reflexión crítica orientada al cambio.

Familiarizarse con las obras críticas sobre la materia: la aportación de la crítica fílmica feminista es muy importante, compleja y motivadora, por lo que sigue siendo asombroso que estas reflexiones no formen parte de las bibliografías de materias específicas sobre cine. La teoría fílmica canónica, a partir de ellas, es revisada, subvertida y puesta en cuestión de una forma que modifica totalmente la ciencia que había sido considerada neutra, universal y uniforme hasta entonces. El alumnado debería acceder a textos imprescindibles como son el ya citado de Laura Mulvey o algunas obras de los años noventa del siglo XX entre las que destacan las de Annette Kuhn 
(1991), Teresa de Lauretis (1992) y Giulia Colaizzi (1995). Como se ha reflejado ya, la bibliografía producida desde los Estudios de las Mujeres, Feministas y de Género es muy abundante, incluyendo a autoras españolas recientes, por lo que su lectura es accesible incluso para profesorado sin experiencia en la materia.

\section{Conclusiones}

Tal y como señala la literatura especializada, la asunción de contenidos relativos al género en la universidad española, tras la total implantación del EEES, es deficiente. Aunque todavía existe cierta oferta de estudios de posgrado en muchas universidades, la presencia de asignaturas específicas en los títulos, que había recibido cierto impulso en las extinguidas Licenciaturas y Diplomaturas, ha disminuido con la llegada de los planes de estudio de Grado.

En cuanto a la agregación de cuestiones de género de forma transversal en todas las materias, sigue siendo un asunto pendiente. Probablemente porque el profesorado no ha recibido formación específica, las aportaciones de las mujeres a la disciplina y/o las contribuciones de las académicas no se recogen ni en unidades docentes ni en bibliografías, por lo que se reproduce la invisibilidad de los nombres y contribuciones. Ello revela la ausencia de especialización e incluso el desinterés por añadir nuevos conocimientos al canon.

Lo antedicho explica que se dedique el presente artículo a sugerir las líneas metodológicas que el profesorado debería tener en cuenta para paliar las lagunas existentes. Las sugerencias expuestas se encaminan a facilitar una práctica docente que ayude al profesorado a asumir las responsabilidades individuales y colectivas imprescindibles para la erradicación de la discriminación. En este sentido, es útil recordar que la igualdad siempre es intencional en sociedades como la nuestra, donde se ha alcanzado la igualdad formal: depende del grado de compromiso de cada docente, de la energía que quiera comprometer, de la formación que se esfuerce en mantener al día (y casi siempre al margen de la corriente institucional) y, sobre todo, de la convicción de que la igualdad entre mujeres y hombres es un vergonzoso déficit de nuestras sociedades que las aulas deben contribuir a eliminar.

\section{Bibliografía}

AGUILAR, P. (2004). ¿Somos las mujeres de cine? Prácticas de análisis fílmico. Oviedo: Instituto Asturiano de la Mujer.

ARRANZ, F. et al. (2009). La situación de las mujeres y los hombres en el audiovisual español. Estudios sociológico y legislativo. Madrid: Universidad Complutense de Madrid. 
BOSCH, E. et al. (2011). Incorporación de los contenidos en igualdad de oportunidades y género en grado. Palma de Mallorca: Universidad de las Islas Baleares.

BOSCH, E. y FERRER, V. (2012). "La vieja y la nueva universidad: cambios propuestos desde una perspectiva de género". En: UNIDADE DE IGUALDADE (ed.) (2012). II Xornada de Innovación en Xénero. Docencia e investigación. Vigo: Universidad de Vigo. p. 31-49.

COLAIZZI, G. (1995). Feminismo y teoría filmica. Valencia: Episteme.

DE LAURETIS, T. (1992). Alicia ya no. Feminismo, semiótica, cine. Madrid: Cátedra.

GARCÍA, A. (2009). Clases de cine. Compartir miradas en femenino y en masculino. Madrid: Instituto de la Mujer.

GARCÍA, M. T. (2013). “Son invisibles las mujeres científicas?”. En: Estudios sobre el mensaje periodístico, núm. 19, vol. especial abril, Madrid: Universidad Complutense de Madrid. p. 783-792.

KUHN, A. (1991). Cine de mujeres. Feminismo y cine. Madrid: Cátedra.

LAMAS, M. (1995). "La perspectiva de género". En: La Tarea. Revista de Educación y Cultura, núm. 8, Jalisco (México): SNTE [Consultado: 16-04-2010]. En: http:// www.latarea.com.mex/articu/articu8/lamas8.htm.

MENÉNDEZ, M. I. (2007a). "Claves prácticas para elaborar información desde la perspectiva de género. Aportaciones desde el mainstreaming”. En: PLAZA, J. F. y DELGADO, C. (eds.) (2007). Género y comunicación. Madrid: Editorial Fundamentos. p. 149-168.

(2007b). "Periodismo desde la perspectiva de género o la igualdad como criterio de calidad". En: Género y comunicación, núm. 9, Madrid: AMECO. p. 57-88.

MULVEY, L. (1988). Placer visual y cine narrativo. Valencia: Episteme.

STIMPSON, C. R. (1998). "¿Qué estoy haciendo cuando hago estudios de las mujeres en los años 90?". En: NAVARRO, M. y STIMPSON, C. R. (comps.) (1998). ¿Qué son los estudios de las mujeres? México D.F.: Fondo de Cultura Económica. p. 127-165.

\section{Notas}

1 El presente trabajo se ha desarrollado en el marco del proyecto "Violencia de género y cultura popular: representación y recepción", cofinanciado por el Ministerio de Sanidad, Servicios Sociales e Igualdad del Gobierno de España y el Fondo Social Europeo en el marco de del Programa Operativo "Lucha contra la discriminación" (Referencia 115/12). Además, es un resultado del Grupo de Innovación Docente de la Universidad de Burgos para la Calidad de la Educación Universitaria desde la Perspectiva de Género (GIDCEUPG), de carácter multidisciplinar. 


\section{La autora}

María Isabel Menéndez es Doctora en Filosofía y Licenciada en Periodismo. Profesora en la Universidad de Burgos (Área de Comunicación Audiovisual y Publicidad) es experta en Estudios de las Mujeres, Feministas y de Género. En la actualidad es Vicedecana de la Facultad de Humanidades y Educación, Coordinadora del Grado en Comunicación Audiovisual y Directora del Posgrado: Especialista Universitario en Enfoque de Género por la universidad de Burgos. Su línea de investigación principal se dedica al análisis de la comunicación desde la perspectiva de género, temática sobre la que ha publicado monografías y artículos en revistas científicas como Estudios sobre el Mensaje Periodístico, Communication \& Society/Comunicación y Sociedad o Telos. Cuadernos de comunicación e innovación entre otras. 